\title{
Effects of elastic strain energy and interfacial stress on the equilibrium morphology of misfit particles in heterogeneous solids
}

\author{
Xujun Zhao ${ }^{1}$, Ravindra Duddu ${ }^{3}$, Stéphane P.A. Bordas ${ }^{4}$ and Jianmin $\mathrm{Qu}^{1,2 *}$ \\ ${ }^{1}$ Department of Mechanical Engineering, Northwestern University, Evanston, IL USA 60208. \\ ${ }^{2}$ Department of Civil and Environmental Engineering, Northwestern University, Evanston, IL \\ USA 60208. \\ ${ }^{3}$ Department of Civil and Environmental Engineering, Vanderbilt University, Nashville, TN \\ USA 37235. \\ ${ }^{4}$ Cardiff School of Engineering, Institute of Mechanics and Advanced Materials Theoretical, \\ Applied and Computational Mechanics, Cardiff University, The Parade, Cardiff CF24 3AA, \\ Wales, UK.
}

\begin{abstract}
This paper presents an efficient sharp interface model to study the morphological transformations of misfit particles in phase separated alloys. Both the elastic anisotropy and interfacial energy are considered. The geometry of the material interface is implicitly described by the level set method so that the complex morphological transformation of microstructures can be accurately captured. A smoothed extended finite element method is adopted to evaluate the elastic field without requiring remeshing. The equilibrium morphologies of particles are shown to depend on the elastic anisotropy, interfacial energy as well as the particle size. Various morphological transformations, such as shape changes from spheres to cuboids, directional aligned platelets and particle splitting, are observed. The simulated results are in good agreement with experimental observations. The proposed model provides a useful tool in understanding the morphological transformation of precipitates, which will facilitate the analysis and design of metallic alloys.
\end{abstract}

Keywords: Morphological transformation; Particle splitting; Equilibrium shape; Extended finite element method; Level set method;

* corresponding author: j-qu@northwestern.edu 


\section{Introduction}

Since the macroscopic properties of heterogeneous materials are closely related to their underlying microstructures, morphologies of the microstructures in heterogeneous materials have been of great interest for material design and constitutive modeling. In particular, new types of precipitate strengthened alloys with desired mechanical properties can be designed by controlling the morphologies of precipitates. Many different morphological transformations have been reported in a number of phase-separated alloys. For example, it was experimentally observed that second phase particles in Ni-based superalloys undergo shape changes from spheres to cuboids with round corners, to platelets aligned along crystallographic directions when the superalloys are annealed at some specific temperature below the precipitation line (Fahrmann et al., 1995; Maheshwari and Ardell, 1993). In addition, more complex phenomena of morphological transformations, such as splitting of a single large particle into several small ones and coalescence of small particles to form highly non-equiaxed plates, have also been found in experiments (Doi et al., 1984; Ma and Ardell, 2007; Miyazaki et al., 1982). Therefore, in order to design new alloys with stable material properties, it is desirable to understand the equilibrium morphologies of microstructures at different length scales.

It has been experimentally demonstrated that both the elastic strain energy and the interfacial energy play important roles on the microstructural morphologies (Conley et al., 1989; Fahrmann et al., 1995). The former is caused by the lattice misfitting between the precipitate and matrix and the latter is due to the existence of particle-matrix interfaces. From the thermodynamic point of view, the equilibrium morphology of particles in this elastically constrained system is achieved by minimizing the total system free energy, i.e. the sum of elastic strain energy and interfacial energy. As a result, the elastic constants and anisotropy of the two phases involved, as well as the interfacial quantities, will affect the equilibrium morphology. For a detailed review, the reader is referred to (Doi, 1996). It should be mentioned that the interfacial energy for solid materials is generally deformation dependent rather 
than constant, which differs from that of liquids (Cammarata and Sieradzki, 1994; Dingreville et al., 2005).

The difficulties in modeling microstructure evolution in phase separated alloys stem from the facts that (i) material properties are discontinuous across the precipitate-matrix interface; and (ii) topological changes of interface during the transformation are difficult to capture numerically. General approaches for simulating the morphological development of particles can be broadly classified on the basis of how the interface is resolved, and are known as diffuse interface and sharp interface models; and how the interface is represented: Lagrangian and Eulerian descriptions.

The diffuse interface model, using an Eulerian description (e.g. phase field method) describes the geometrical interface utilizing an order parameter that smoothly changes across the interface so that the location of the interface is not tracked. This approach is advantageous in simulating microstructural evolution with interaction of multiple particles and topological changes, such as particle coalescence and splitting ( $\mathrm{Hu}$ and Chen, 2001; Lou and Bassani, 2008; Wang et al., 1993). Nevertheless, the main drawback of such diffuse interface models is that their computations are very complex, because the diffuse interface must be resolved by using a grid spacing smaller than the interface thickness to ensure accuracy (Provatas and Elder, 2010). Typically, in real materials, the particle-matrix interface thickness is infinitesimally small compared to the particle size, which necessitates a very refined mesh. Furthermore, the parameters involved in the phase field equations are usually difficult to determine for realistic materials.

By contrast, the sharp interface approaches use a dividing interface to distinguish the two separate phases. For example, the equilibrium shapes of misfitting particles in an elastically anisotropic system have been formulated based on the boundary integral methods (BIM), in which the interface was explicitly tracked by marker particles (Schmidt and Gross, 1997; Schmidt et al., 1998; Su and Voorhees, 1996; Voorhees et 
al., 1992). Jog et al. (Jog et al., 2000) proposed a general approach for determining the equilibrium shape of isolated misfitting particles by minimizing the sum of elastic and interfacial energy using a combination of finite element method (FEM) and an optimization technique. However, for the conventional BIM and FEM based approaches using a Lagrangian particle tracking method, a set of marker points have to be placed on the interfaces to track its motion so it is extremely difficult to handle topological changes such as particle merging and breaking, which have been extensively observed in actual experiments (Doi et al., 1984; Kaufman et al., 1989; Miyazaki et al., 1982).

The present work is to study the equilibrium morphologies of inhomogeneities in a generally anisotropic elastic solid with arbitrary misfit strains without any a priori assumption of the possible precipitate morphologies. The interfacial energy is assumed deformation-dependent and is taken into account by using the Gurtin and Murdoch interface elasticity model (Gurtin and Murdoch, 1975). We employ a sharp interface model based on a hybrid extended finite element and level set method that naturally allows complex topological changes during the morphological evolution without requiring remeshing. The level set method, originally devised for tracking moving interfaces (Osher and Sethian, 1988), is capable of describing complicated geometrical interfaces of microstructures, such as dendritic solidification (Chen et al., 1997; Zabaras et al., 2006), precipitate evolution (Duddu et al., 2011) and biofilm growth (Duddu et al., 2008; Duddu et al., 2009).. The combined method enables the handling of complex morphological transformations of multiple misfitting inhomogeneities. The extended finite element method (XFEM) (Moës et al., 2003; Moës et al., 1999) is used to compute the elastic field of the particle-matrix system with the interface elasticity effect. The main advantage of XFEM is that the meshes are not required to conform with the geometrical interface, so that remeshing is not needed when the interface moves. The equilibrium morphologies of particles are obtained by minimizing the sum of elastic strain energy and interfacial energy at a constant total mass of the particles. We show that the complex morphological 
transformations observed in experiments, such as splitting and merging, can be accurately captured for different particle sizes.

\section{Problem formulation}

We consider a generally anisotropic elastic solid $V$ containing a number of inhomogeneous particles with arbitrary eigenstrains. Assume the particles occupy domain $\Omega$ with arbitrary shape bounded by the smooth interface $\Gamma$. The particle-matrix interfaces are assumed to be coherent and the external boundary of $V$ is subjected to traction-free boundary conditions. In the absence of body force, the governing equations of the elastic bulk materials are as follows:

$$
\begin{aligned}
& \nabla \cdot \boldsymbol{\sigma}=0 \\
& \boldsymbol{\sigma}=\mathbf{C}(\mathbf{x}): \mathbf{e}(\mathbf{x})= \begin{cases}\mathbf{C}^{\mathbf{I}}:\left[\boldsymbol{\varepsilon}(\mathbf{x})-\boldsymbol{\varepsilon}^{*}\right] & \mathbf{x} \in \Omega \\
\mathbf{C}^{\mathbf{M}}: \boldsymbol{\varepsilon}(\mathbf{x}) & \mathbf{x} \notin \Omega\end{cases} \\
& \boldsymbol{\varepsilon}=\frac{1}{2}\left[\nabla \mathbf{u}+(\nabla \mathbf{u})^{T}\right]
\end{aligned}
$$

where $\mathbf{u}$ denotes the displacement vector, $\boldsymbol{\sigma}$ and $\boldsymbol{\varepsilon}$ are the stress and strain tensors, respectively, $\mathbf{e}$ is the elastic strain tensor, $\boldsymbol{\varepsilon}^{*}$ is the eigenstrain tensor that measures the lattice misfit between the particle and matrix, and $\mathbf{C}^{\mathbf{I}}$ and $\mathbf{C}^{\mathbf{M}}$ represent the elastic stiffness tensors of inhomogeneity and matrix respectively.

Due to the existence of interfacial stress, the mechanical equilibrium conditions on the interface are governed by the generalized Young-Laplace equation (Gurtin and Murdoch, 1975; Povstenko, 1993)

$$
\llbracket \boldsymbol{\sigma} \rrbracket \cdot \mathbf{n}+\nabla \cdot \boldsymbol{\sigma}^{S}=0
$$

where $\llbracket \cdot \rrbracket=(\cdot)^{\text {out }}-(\cdot)^{\text {in }}$ represents the quantity jump across the interface, $\mathbf{n}$ is outward normal, and $\boldsymbol{\sigma}^{S}$ is interfacial stress. The displacement across the particle-matrix interface of coherent particles is continuous, i.e.

$$
\llbracket \mathbf{u} \rrbracket=0
$$


The total system free energy is given by the sum of the elastic strain energy and the interfacial energy

$$
F=\int_{V} W d V+\int_{\Gamma} \gamma d S
$$

where $W=\frac{1}{2} \boldsymbol{\sigma}: \mathbf{e}$ is the elastic strain energy density, and $\gamma$ is the interfacial energy density, which can be written as (Dingreville et al., 2005)

$$
\gamma=\gamma_{0}+\boldsymbol{\tau}^{S}: \boldsymbol{\varepsilon}^{S}+\frac{1}{2} \boldsymbol{\varepsilon}^{S}: \mathbf{C}^{S}: \boldsymbol{\varepsilon}^{s}
$$

Field quantities with a superscript $S$ in the above equation are associated with the interfaces. For example, $\boldsymbol{\varepsilon}^{S}$ is the interface strain tensor, $\mathbf{C}^{S}$ is the interface elastic stiffness tensor, $\boldsymbol{\tau}^{s}$ is the interface residual stress, and $\gamma_{0}$ is the interfacial free energy density that corresponds to the interfacial energy state when $\boldsymbol{\varepsilon}^{S}=\mathbf{0}$. The interfacial strain can be computed from

$$
\boldsymbol{\varepsilon}^{s}=\frac{1}{2}\left[\mathbf{P} \cdot\left(\nabla_{s} \mathbf{u}\right)+\left(\nabla_{s} \mathbf{u}\right)^{T} \cdot \mathbf{P}\right]
$$

where $\mathbf{P}=\mathbf{I}-\mathbf{n} \otimes \mathbf{n}$ is the projection tensor, $\mathbf{I}$ is the identity tensor, $\otimes$ represents a dyad, and the surface gradient is defined as $\nabla_{s} \mathbf{u}=\nabla \mathbf{u} \cdot \mathbf{P}$. Consequently, the corresponding interfacial stress can be obtained from the Shuttleworth equation (Shuttleworth, 1950),

$$
\boldsymbol{\sigma}^{S}=\frac{\partial \gamma}{\partial \boldsymbol{\varepsilon}^{S}}=\boldsymbol{\tau}^{S}+\mathbf{C}^{S}: \boldsymbol{\varepsilon}^{S}
$$

In the early stage of phase decomposition, a new phase nucleates from the matrix and grows by absorbing the supersaturated solute atoms from its neighboring substrate until the solute concentration in the matrix decreases to the equilibrium state. When the formation of a new phase is finished, the matrix can no longer provide solute atoms to the precipitates. Therefore, the mass fraction remains constant during the subsequent aging, although the morphology of particles will continue to change to adjust the total system energy. During this process, the chemical free energy can be reasonably assumed to remain constant. Therefore, the total system energy is the sum of the elastic energy and interfacial energy, and the equilibrium morphology depends on the balance of elastic energy and interfacial energy (Doi, 1996). Based on the above assumptions, the problem can be modeled by minimizing the total energy under the constraint that the mass fraction remains constant, which is equivalent to the 
constraint that the volume fraction remains constant for the small deformation considered here. As a result, the energy functional $F$ can be modified by incorporating a Lagrangian multiplier

$$
\bar{F}=\int_{V} W d V+\int_{\Gamma} \gamma d S+\lambda\left(\int_{\Omega} d V-V_{0}\right)
$$

where $V_{0}$ is the prescribed total volume of particles. It has been shown that the Lagrangian multiplier $\lambda$ can be identified as a chemical driving force, which is the jump of chemical free energy density across the interface (Schmidt and Gross, 1997).

Therefore, the present problem becomes a minimization problem, i.e., searching a minimum of energy functional $\bar{F}$ subjected to the mechanical equilibrium equations (1)-(5).

\section{Kinetics of the interface}

To derive the driving force on the interface, let us introduce a fictitious time $t$. In continuum mechanics, the material derivative of a quantity is usually denoted as $D($ ) / Dt consisting of two parts: the first part consists of the local rate of the quantity change at a fixed material point and the second part is related to changes due to variation of the domain itself. For example, the material derivative of the displacement vector can be written as

$$
\frac{D \mathbf{u}}{D t}=\frac{\partial \mathbf{u}}{\partial t}+(\nabla \mathbf{u}) \cdot \mathbf{v}
$$

where $\mathbf{v}=d \mathbf{x} / d t$ is the velocity vector. For simplicity, we use 'over dot' and a 'prime' to represent the material derivative and local derivative, respectively, which leads to

$$
\dot{\mathbf{u}}=\mathbf{u}^{\prime}+(\nabla \mathbf{u}) \cdot \mathbf{v}
$$

According to the Reynolds transport theorem (Belytschko et al., 2000), taking the material derivative of the elastic energy functional yields

$$
\begin{aligned}
\frac{D}{D t} \int_{V} W d V & =\int_{V}\left[\frac{\partial W}{\partial t}+\operatorname{div}(W \mathbf{v})\right] d V \\
& =\int_{V}[\boldsymbol{\sigma}: \nabla \dot{\mathbf{u}}-\boldsymbol{\sigma}: \nabla(\nabla \mathbf{u} \cdot \mathbf{v})+\operatorname{div}(W \mathbf{v})] d V
\end{aligned}
$$


Using the Green's theorem, the above equation becomes

$$
\frac{D}{D t} \int_{V} W d V=\int_{V} \boldsymbol{\sigma}: \nabla \dot{\mathbf{u}} d V-\int_{\Gamma} \mathbf{n} \cdot \llbracket \mathbf{\Sigma} \rrbracket \cdot \mathbf{v} d S
$$

where

$$
\boldsymbol{\Sigma}=W \mathbf{I}-\boldsymbol{\sigma} \cdot \nabla \mathbf{u}
$$

is Eshelby’s elastic energy momentum tensor (Eshelby, 1975).

The material derivative of the interfacial energy density gives rise to

$$
\begin{aligned}
\frac{D}{D t} \int_{V} \gamma d V & =\int_{\Gamma}\left(\dot{\gamma}-\gamma \kappa v_{n}\right) d S \\
& =\int_{\Gamma} \boldsymbol{\sigma}^{S}: \nabla_{S} \dot{\mathbf{u}} d S-\int_{\Gamma} \gamma \kappa v_{n} d S
\end{aligned}
$$

where $v_{n}=\mathbf{v} \cdot \mathbf{n}$ is the normal velocity and $\kappa$ is the interface mean curvature. The material derivative of the volume integral is straightforward,

$$
\frac{D}{D t} \int_{V} d V=\int_{\Gamma} v_{n} d S
$$

Therefore, the material derivative of the energy functional (10), by using equations (15), (16) and (17), can be easily obtained as follows:

$$
\frac{D \bar{F}}{D t}=\int_{V} \boldsymbol{\sigma}: \nabla \dot{\mathbf{u}} d V+\int_{\Gamma} \boldsymbol{\sigma}^{S}: \nabla_{S} \dot{\mathbf{u}} d S-\int_{\Gamma}(\mathbf{n} \cdot \llbracket \boldsymbol{\Sigma} \rrbracket \cdot \mathbf{n}+\gamma \kappa-\lambda) v_{n} d S
$$

Since $\dot{\mathbf{u}}$ is an arbitrary variation which can be treated as a test function that $\dot{\mathbf{u}}=\delta \mathbf{u}$, it is obvious that the first two integrals of above equation are the weak forms of equilibrium equations (1)-(5), and should vanish when the system arrives at mechanical equilibrium. This can be illustrated as follows:

$$
\begin{aligned}
\int_{V} \boldsymbol{\sigma}: \nabla \dot{\mathbf{u}} d V & +\int_{\Gamma} \boldsymbol{\sigma}^{S}: \nabla_{S} \dot{\mathbf{u}} d S \\
& =\int_{V} \nabla \cdot(\boldsymbol{\sigma} \cdot \dot{\mathbf{u}})-(\nabla \cdot \boldsymbol{\sigma}) \cdot \dot{\mathbf{u}} d V+\int_{\Gamma} \nabla_{S} \cdot\left(\boldsymbol{\sigma}^{S} \cdot \dot{\mathbf{u}}\right)-\left(\nabla_{S} \cdot \boldsymbol{\sigma}^{S}\right) \cdot \dot{\mathbf{u}} d S \\
& =-\int_{V}(\nabla \cdot \boldsymbol{\sigma}) \cdot \dot{\mathbf{u}} d V-\int_{\Gamma}\left(\llbracket \boldsymbol{\sigma} \rrbracket \cdot \mathbf{n}+\nabla_{S} \cdot \boldsymbol{\sigma}^{S}\right) \cdot \dot{\mathbf{u}} d S \\
& =0
\end{aligned}
$$

Consequently, equation (18) reduces to

$$
\frac{D \bar{F}}{D t}=-\int_{\Gamma}(\mathbf{n} \cdot \llbracket \boldsymbol{\Sigma} \rrbracket \cdot \mathbf{n}+\gamma \kappa-\lambda) v_{n} d S
$$

If we take 


$$
v_{n}=\mathbf{n} \cdot \llbracket \Sigma \rrbracket \cdot \mathbf{n}+\gamma \kappa-\lambda
$$

then equation (20) becomes

$$
\frac{D \bar{F}}{D t}=-\int_{\Gamma} v_{n}^{2} d S \leq 0
$$

In other words, (21) guarantees that the total system energy always decreases in the steepest decent direction during the interface evolution. It should be mentioned that the normal velocity (21) is of the same form as the necessary equilibrium condition in reference (Schmidt and Gross, 1997). However, our work differs from theirs in two respects. First, the interfacial energy $\gamma$ in the present work is deformation dependent, which is related to interfacial residual stress and interfacial elasticity. Similarly, the energy momentum tensor $\boldsymbol{\Sigma}$ is also affected by the interfacial stress due to the coupled boundary condition (4). Second, it is possible that the necessary equilibrium condition in (Schmidt and Gross, 1997) may lead to a maximum or a saddle point unless the sign of the second variation of energy functional is considered. In contrast, our formulation guarantees that the system always evolves in a direction that decreases the total system energy, so that at least a local minimum is achieved.

Finally, the Lagrangian multiplier can be obtained from the mass conservation condition, i.e. $\frac{D}{D t} \int_{V} d V=0$. Substituting (21) into (17) yields

$$
\int_{\Gamma}(\mathbf{n} \cdot \llbracket \Sigma \rrbracket \cdot \mathbf{n}+\gamma \kappa-\lambda) d S=0
$$

Hence, we have

$$
\lambda=\frac{\int_{\Gamma}(\mathbf{n} \cdot \llbracket \mathbf{\Sigma} \rrbracket \cdot \mathbf{n}+\gamma \kappa) d S}{\int_{\Gamma} d S}
$$

\section{Level set description of implicit interfaces}

In practice, complex topological changes take place during the morphological evolution of single or multiple particles, such as merging and splitting. It is therefore desirable to describe the geometrical interfaces without an explicit parameterization. 
Moreover, it is also desirable that the method is capable of dealing with multiple particles within a uniform framework.

To meet these needs, we choose the level set method (LSM) for an implicit representation of the particle interface. LSM was originally devised to capture the propagating fronts (Osher and Sethian, 1988) and quickly led to a wide range of applications for tracking moving interface. In this method, the propagating interface is embedded as the zero level set of a higher dimensional function $\phi(\mathbf{x}, t)$ with its initial value given by

$$
\phi(\mathbf{x}, t=0)= \pm d
$$

where $\pm d$ is the signed distance function to the interface, such that $\phi<0$ in the particles, $\phi>0$ in the matrix and $\phi=0$ on the interface. A typical level set function is shown in Fig. 1.

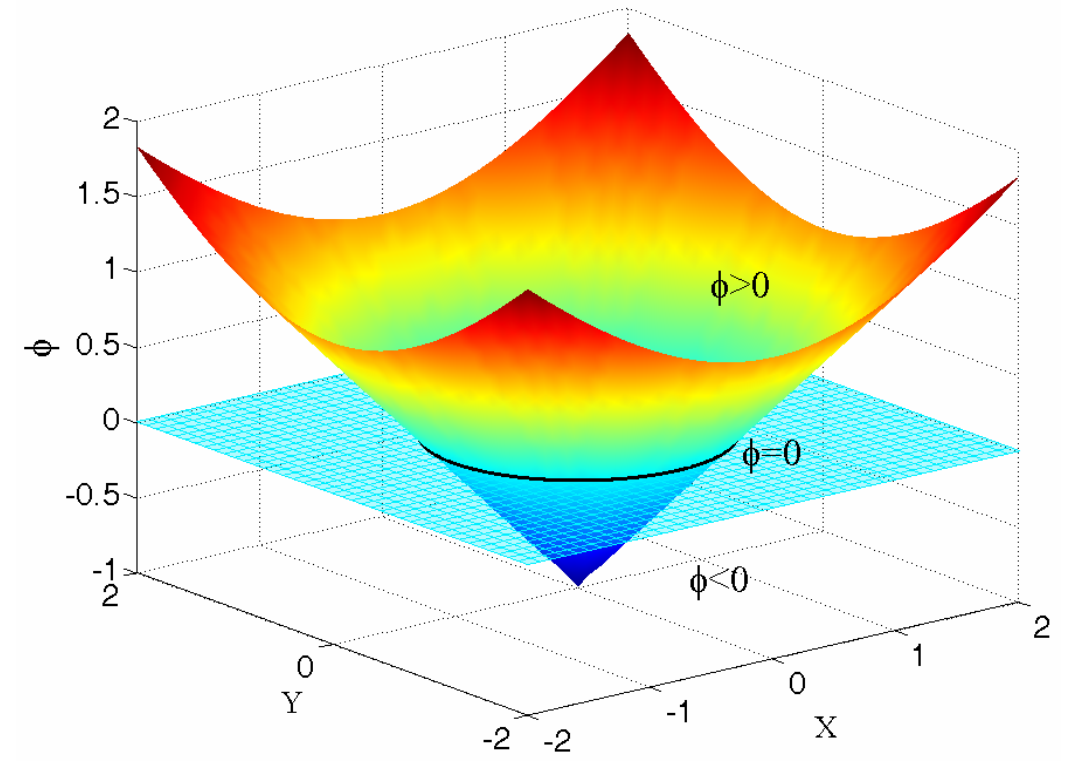

Fig. 1 Level set function.

The geometric quantities such as the normal vector $\mathbf{n}$ and the curvature $\kappa$ can be conveniently expressed in terms of the level set function as 


$$
\mathbf{n}=\frac{\nabla \phi}{|\nabla \phi|}, \quad \kappa=\nabla \cdot \frac{\nabla \phi}{|\nabla \phi|}
$$

An initial boundary value partial differential equation then can be obtained for the evolution of $\phi$ by taking a material derivative on both sides of (25)

$$
\frac{\partial \phi}{\partial t}+\mathbf{v}^{e x t} \cdot \nabla \phi=0 \quad \text { or } \quad \frac{\partial \phi}{\partial t}+v_{n}^{e x t}|\nabla \phi|=0
$$

which is well known as the level set evolution equation. The position of the interface at any time $t$ is given by the zero level set $\phi(\mathbf{x}, t)=0$. Now the equilibrium morphology problem discussed in the previous section becomes a problem of finding a scalar function $\phi$ that minimizes the system energy functional $\bar{F}$.

It is noted that the velocity field $\mathbf{v}^{\text {ext }}$ or $v_{n}^{\text {ext }}$ in the level set equation is defined in the entire computational domain, while the velocity given in (21) is only defined on the interface. Therefore, a velocity extension should be conducted to construct the velocity field from the interface to the whole computational domain. In this work, a second order fast marching method (Sethian, 1996, 1999a) is adopted to construct the spatial velocity $v_{n}^{e x t}$ that satisfies the equation

$$
\nabla v_{n}^{e x t} \cdot \nabla \phi=0
$$

This algorithm allows the interface to move under a velocity that maintains the signed distance function, so that no re-initialization process is required during the evolution of the interface (Adalsteinsson and Sethian, 1999; Sethian, 1999a).

The level set equation (27) is a hyperbolic partial differential equation (PDE), hence numerical schemes designed for hyperbolic conservation laws can be used to produce stable solutions. Here we use the robust third order accurate Hamilton-Jacobi WENO finite difference spatial discretization (Liu et al., 1994) and the third order TVD Runge-Kutta (RK) time discretization schemes (Shu and Osher, 1988) to solve this hyperbolic PDE. In addition, the time step $\Delta t$ must be carefully chosen to ensure the 
time stability of upwind scheme. The Courant-Friedrichs-Lewy (CFL) condition requires $\Delta t$ to satisfy

$$
\Delta t<\alpha \frac{h}{\max \left(\left|v_{n}^{e x t}\right|\right)}
$$

where $h$ is the grid size and $0<\alpha<1$. For a detailed discussion of level set method and its numerical strategies, the readers are referred to the corresponding literatures (Osher and Fedkiw, 2003; Sethian, 1999b).

\section{Smoothed extended finite element method}

One of the key issues in the level set update of interface is to calculate the elastic field in order to obtain the interface velocity given in equation (21). However, the standard FEM requires the finite element mesh to coincide with the material interface so as to maintain the accuracy and optimal convergence, so that remeshing has to be performed when the interface moves, especially with significant topological changes. In contrast, the extended finite element method (XFEM) locally modifies the shape functions of the standard FEM by introducing an additional enrichment part and it is allowed to model particular discontinuities or singularities without requiring the mesh to conform with the geometrical interfaces (Belytschko et al., 2001; Moës et al., 1999), which provides great convenience for moving interface problems. An open source C++ XFEM code is also available for use (Bordas et al., 2007). In the present study, a modified XFEM with strain smoothing technique called SmXFEM (Zhao et al., 2012) was adopted to compute the elastic field in the presence of interface energy effect. Here we give a brief introduction about the computational methodology.

The weak form of the equilibrium equation (19) can be rewritten into the following form by letting $\dot{\mathbf{u}}=\delta \mathbf{u}$,

$$
\int_{V} \boldsymbol{\sigma}(\mathbf{u}): \boldsymbol{\varepsilon}(\delta \mathbf{u}) d V+\int_{\Gamma} \boldsymbol{\sigma}^{S}(\mathbf{u}): \boldsymbol{\varepsilon}^{S}(\delta \mathbf{u}) d S=0
$$

In the inhomogeneity-matrix problem, the displacement in the finite element can be approximated in the following form: 


$$
\mathbf{u}^{h}(\mathbf{x})=\mathbf{N}(\mathbf{x}) \mathbf{d}+F(\mathbf{x}) \mathbf{N}(\mathbf{x}) \mathbf{a}
$$

where $\mathbf{d}$ and a correspond to the nodal displacement vector and additional degrees of freedom due to enrichments, respectively, $\mathbf{N}(\mathbf{x})$ is the standard finite element shape function matrix, and $F(\mathbf{x})$ is the enrichment function given by (Moës et al., 2003)

$$
F(\mathbf{x})=\sum_{I} N_{I}(\mathbf{x})\left|\phi_{I}\right|-\left|\sum_{I} N_{I}(\mathbf{x}) \phi_{I}\right|
$$

where $\phi_{I}$ is the value of level set function at node $I$. It is easily seen that in a standard finite element that does not contain any part of interface, the enrichment function $F(\mathbf{x})$ vanishes and (31) reduces to the displacement field in the standard FEM formulation. In other words, only the elements that are traversed by the interface are enriched with extra degrees of freedom.

Different from the conventional XFEM, a strain smoothing technique (Liu et al., 2007) is used to construct the strain field in the present finite element formulation. The basic idea is that each finite element is divided into several smoothing cells and the strain field is assumed to be uniform in each of these smoothing cells. The smoothed strain in the $k$-th smoothing cell can be expressed as

$$
\overline{\boldsymbol{\varepsilon}}\left(\mathbf{x}_{k}\right)=\frac{1}{A_{k}^{s}} \int_{\Gamma_{k}^{s}} \mathbf{L}_{k}(\mathbf{x}) \cdot \mathbf{u}^{h}(\mathbf{x}) d \Gamma
$$

where $A_{k}^{s}$ is the area of the smoothing cell $\Omega_{k}^{s}$, and $\mathbf{L}_{k}(\mathbf{x})$ is a matrix function of the outward normal vector on the boundary $\Gamma_{k}^{s}$ of smoothing cell $\Omega_{k}^{s}$ that has the form

$$
\mathbf{L}_{k}(\mathbf{x})=\left[\begin{array}{cc}
n_{x} & 0 \\
0 & n_{y} \\
n_{y} & n_{x}
\end{array}\right] .
$$

It can be seen that in such a way, the domain integral over $\Omega_{k}^{s}$ is transformed into a 
boundary integral along the edges of the smoothing cells and the evaluation of the Jacobian in each element can be avoided. Also, we can see that the displacement gradient does not appear in equation (33). As a result the derivatives of the shape functions are not required in the smoothed finite element method. These advantages could effectively improve the computational efficiency without loss of accuracy of the XFEM at the same time. The readers are referred to the paper (Zhao et al., 2012) and the cited references therein for the detailed numerical implementation of SmXFEM.

\section{Results and discussion}

This section provides a variety of numerical examples and validations through comparison with observed experimental results. Although the formulations derived above are general and suitable for arbitrary anisotropy in material symmetries and misfit strains in both the inhomogeneity and the matrix, we will limit our examples to two dimensions and mainly focusing on the Nickel-base alloys that have the cubic symmetry and dilatational misfit eigenstrains. We use the thermodynamic data of the $\gamma-\gamma^{\prime}$ Ni-alloys for $\mathrm{Ni}_{3} \mathrm{Al}$ precipitates and $\mathrm{Ni}-\mathrm{Al}$ matrix, which were determined experimentally in (Fahrmann et al., 1999). The elastic constants are $C_{11}^{I}=179 \mathrm{GPa}$, $C_{12}^{I}=123 \mathrm{GPa}$ and $C_{44}^{I}=81 \mathrm{GPa}$ for the inhomogeneities, and $C_{11}^{M}=161 \mathrm{GPa}$, $C_{12}^{M}=107 \mathrm{GPa}$ and $C_{44}^{M}=85 \mathrm{GPa}$ for the matrix. The lattice misfit is $+0.28 \%$ (Fahrmann et al., 1999). We assume the interface is isotropic and the interfacial free energy is $\gamma_{0}=0.0142$ (Ardell, 1968). For two-dimensional problems, the interface residual $\boldsymbol{\tau}^{S}$ and stiffness constants $\mathbf{C}^{S}$ are scalars and will be expressed by $\tau^{S}$ and $\kappa^{S}$, respectively. Consequently, we can introduce the dimensionless characteristic length $L=C_{44}^{I} \varepsilon_{0}^{2} R_{0} / \gamma_{0}$, where $R_{0}$ is the effective radius of particles, for example, $R_{0}=\sqrt{A_{0} / \pi}$ where $A_{0}$ is the area of a particle in two-dimensions. The simulation domain size is $12 \times 12 R_{0}^{2}$, unless otherwise specified. In addition, the material axis $<100>$ directions are assumed to be the same as the spatial coordinate 
directions.

\subsection{Equilibrium shape of a single particle}

The equilibrium shape of an isolated particle has been studied by many researchers (Jog et al., 2000; Schmidt and Gross, 1997; Voorhees et al., 1992), so it provides a good benchmark problem for our numerical computations. In this example, we use a regular quadrilateral mesh with $182 \times 182$ elements in the simulation. For small particles, the interfacial energy prevails and the equilibrium shape is closed to a circle. With the increase of particle size, the elastic energy becomes important and gradually predominates over the interfacial energy. Finally, it governs the equilibrium shape of particles, which is square-like for cubic crystals.

For simplicity, we firstly assume $\tau^{S}=0$ and $\kappa^{S}=0$, so the interfacial energy becomes a constant. Fig. 2 shows the total system energy evolution curves for single particles of different sizes. The initial particle shape is assumed as a circle, which is to represent the initial stage of a coarsening process. The total energy is normalized by the reference energy value at the initial configuration. It is noticed that the total system energy keeps decreasing during the evolution process until the system arrives at an equilibrium state, then the energy becomes constant and the morphology stops changing in the subsequent iterations. It is also noted that the energy change from a circular particle to a square-like particle is rather small, for example, about $3 \%$ for $L=$ 20. However, this small change does have a great effect on the particle morphology. 


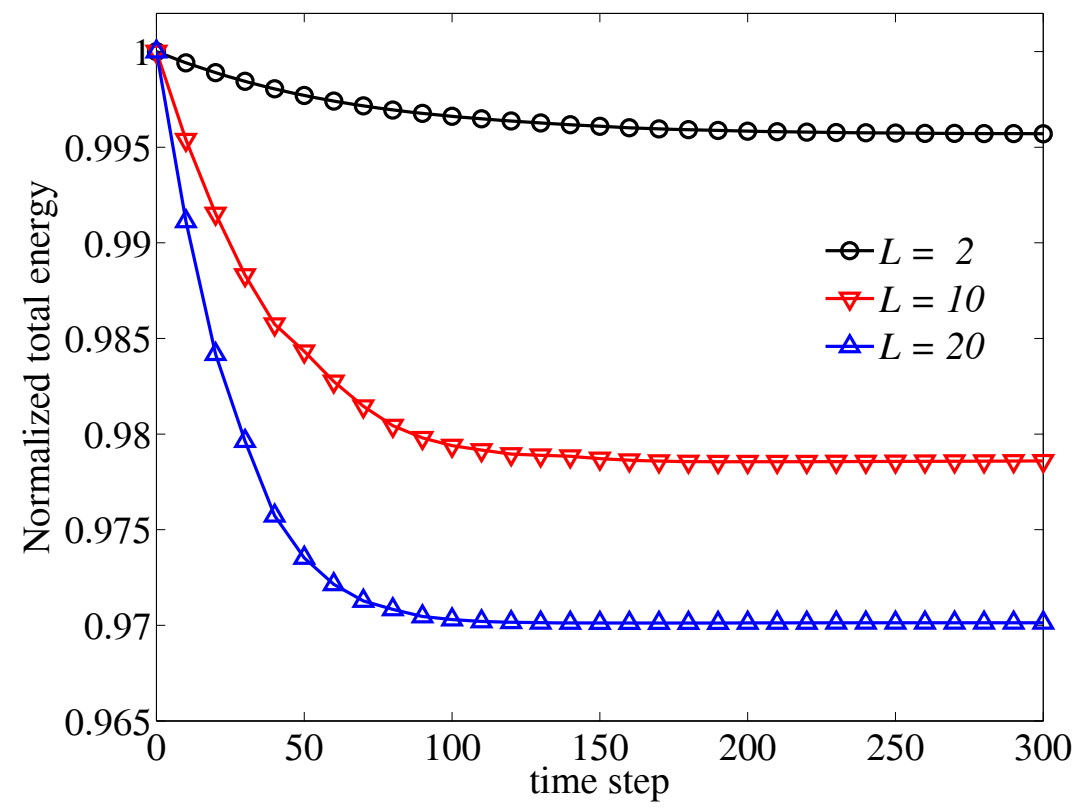

Fig. 2 Normalized total system energy vs. time steps for particles with different sizes.

The final equilibrium morphologies of particles for various sizes are given in Fig. 3. It is clearly seen that the equilibrium morphologies of particles transform from circle-like to square-like shapes with the increase of the particle size, and flat edges form along the elastically soft $<100>$ directions. These results are in good agreement with both numerical simulation and experimental observations (Fahrmann et al., 1995; Schmidt and Gross, 1997; Voorhees et al., 1992). A temporal evolution process of an initially circular particle is shown in Fig. 4, in which a circular particle gradually changes into a four-fold symmetric square-like shape in appearance due to the bulk elastic energy. It can be seen that the morphology at the time step $t=200$ is exactly the same as that at $t=300$, which is in accordance with the energy curves in Fig. 2 . Here we note that the variable $t$ is a factitious non-dimensional time, used here to track the steps in the numerical simulations. 


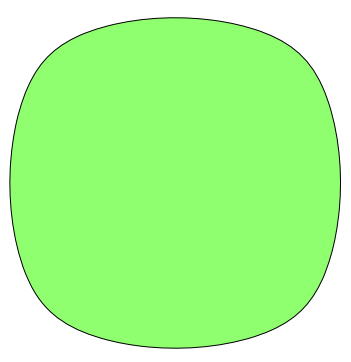

(a)

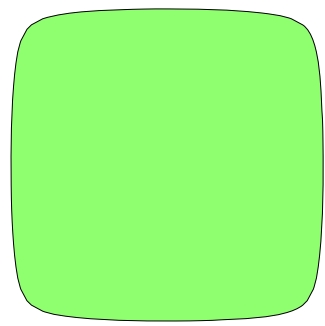

(b)

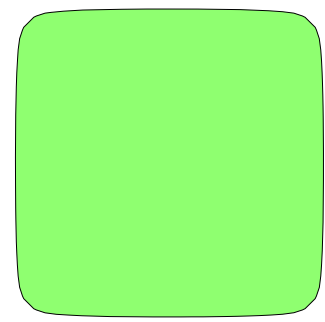

(c)

Fig. 3 Equilibrium shapes of an isolated particle with dilatational eigenstrain at different length scales $\tau^{S}=0$ and $\kappa^{S}=0$. (a) $L=2$, (b) $L=10$, (c) $L=20$

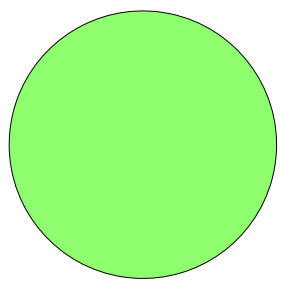

(a)

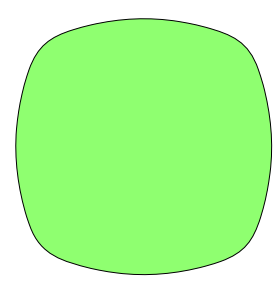

(b)

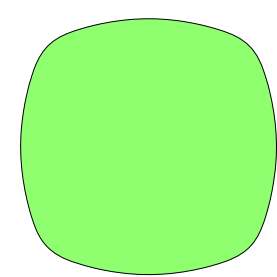

(c)

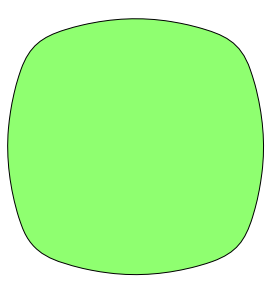

(d)

Fig. 4 Temporal evolution process of an initially circular particle with $L=10$. (a) $t$ $=0$, (b) $t=50$, (c) $t=200$, (d) $t=300$.

In order to investigate the influence of the interfacial residual stress and elasticity, we repeated the calculations for the above example using $\tau^{S}=-1 N / m$ and $\kappa^{S}=10 N / m$ (Tian and Rajapakse, 2007). Fig. 5 and Fig. 6 show the equilibrium shapes of a single particle at different length scales with non-zero interfacial residual stress and interfacial elasticity, respectively. It is seen that their influences on the equilibrium shapes are not significant for the material systems considered here. Therefore, in the following examples, it will be assumed that $\tau^{S}=0$ and $\kappa^{S}=0$ for simplicity. 


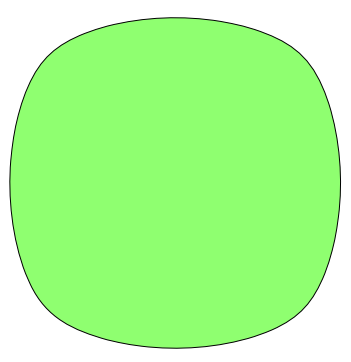

(a)

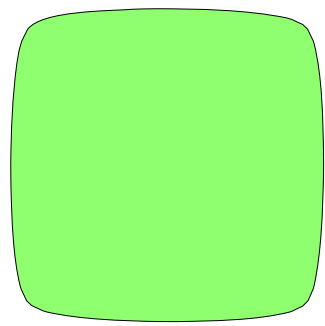

(b)

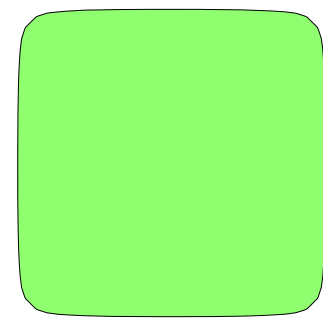

(c)

Fig. 5 Equilibrium shapes of an isolated particle with dilatational eigenstrain at different length scales $\tau^{S}=-1 N / m$ and $\kappa^{S}=0$. (a) $L=2$, (b) $L=10$, (c) $L=20$

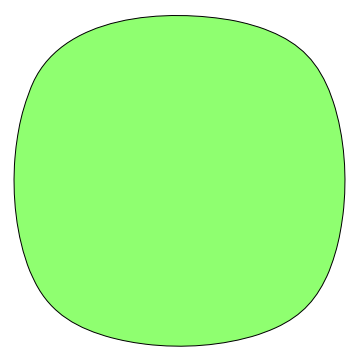

(a)

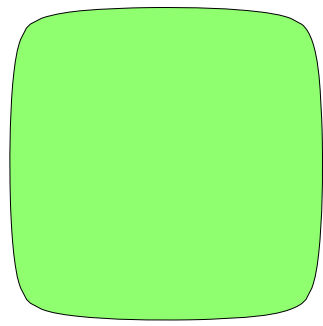

(b)

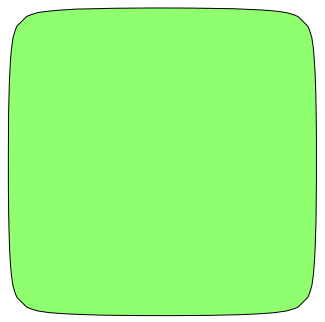

(c)

Fig. 6 Equilibrium shapes of an isolated particle with dilatational eigenstrain at different length scales $\tau^{S}=0$ and $\kappa^{S}=10 N / m$. (a) $L=2$, (b) $L=10$, (c) $L=20$

\subsection{Interaction of multiple particles}

To investigate the effects of particle interaction, we consider a system with two equal sized particles. The initial shape of the two particles is assumed circular and their center-to-center distance is $2.2 R_{0}$. The simulation domain size is $18 \times 12 R_{0}^{2}$, and a regular mesh with $420 \times 280$ elements is adopted.

Fig. 7 shows the morphological changes of the two particles at different time steps. For small particles, it is not surprising that the circle-like shape is maintained due to the predominance of interfacial energy. We can see that the shape change for particles 
$L=2$ is insignificant, especially from time step 500 to 1200 . However, for large particles, the phenomenon becomes more interesting. For example, when $L=20$, the two circular particles firstly change their shapes into square-like ones, during which the adjacent interfaces of two particles continuously move in opposite directions and flat edges are gradually formed, see Fig. 7c (1)-(3). Afterwards, both particles start to elongate in the horizontal direction and the adjacent interfaces move closer and closer to each other until reaching an equilibrium state. For the intermediate sized particles as shown in Fig. 7b, only the square-like shape transformation occurs, but the elongation is not obvious due to the competition between the elastic bulk energy and the interfacial energy.

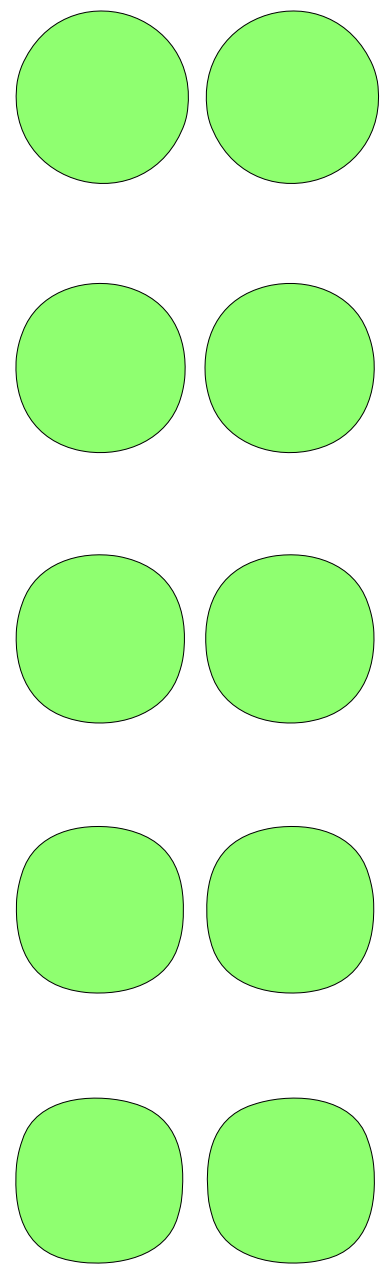

(a) $L=2$
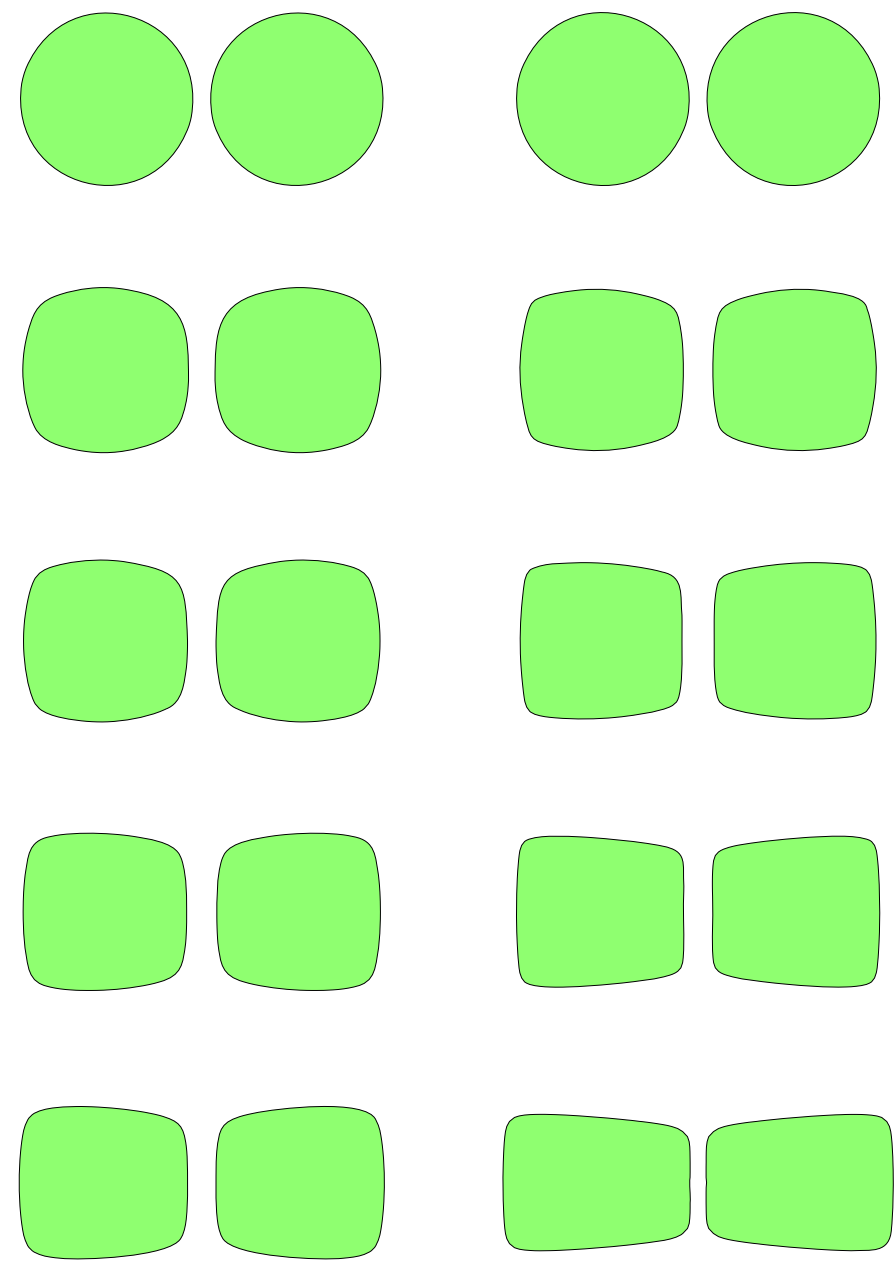

(b) $L=10$

(c) $L=20$

Fig. 7 Temporal morphological changes of a double-particle system under elastic interaction. The time steps are taken as $t=0,100,200,500$ and 1200, respectively. 
It has been theorized that a single four-fold symmetric square-like particle is no longer stable when its size reaches a critical value (Schmidt and Gross, 1997; Thompson et al., 1994). However, this morphology is an equilibrium shape and it will remain unchanged if no external perturbation is introduced, so large square particles sparsely distributed in low volume fraction alloys can usually be observed in experiments (Doi, 1996; Miyazaki et al., 1982), although they are not stable. For high volume fraction alloys where the distance between particles is small, there is a tendency for large particles to align along <100> crystallographic direction, while this directional alignment is not obvious for small particles (Fahrmann et al., 1995). Our numerical results are in qualitative agreement with the experimental observations. This seems to indicate that the elastic interaction between particles plays a central role to facilitate this directional alignment for alloys at high volume fraction.

\subsection{Splitting of precipitate particles}

The splitting phenomenon is usually observed in Ni and Fe-base alloys at low volume fraction, where the coherent $\gamma^{\prime}$ particles sparsely distributed in the matrix can split into a group of small particles during aging at a specific temperature. In such a case, the interfacial energy increases with the creation of new interface, and the elastic energy plays a role that is different from or even opposite to its roles in coarsening, where larger particles grow at the expenses of small particles. Transmission electron microscope (TEM) observations show that the particles undergo complex morphological transformations during splitting. In this section, we will show kinetically how the morphology develops and how the system energy evolves.

Fig. 8 shows the simulated results (the first row) and the comparison with the experimental results for Ni-12at.\%Si alloy (Doi et al., 1984) for particle size $L=50$. We use a four-star shaped particle (Fig. 8a) as the initial configuration to mimic the experimentally observed shape (Fig. 8d). The morphological transformation in this 
process is very complex, and the shape adjustment and splitting proceed simultaneously. It is clearly observed that the concaves at the middle of edges simultaneously extend towards the center of the particle along the elastically soft directions $\{100\}$ and finally split into four small squares with round corners. The simulated results are in excellent agreement with experiments.

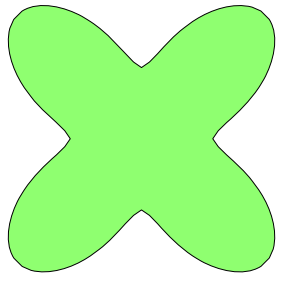

(a)

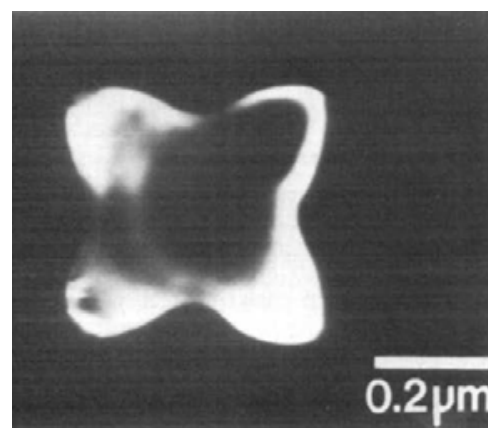

(d)

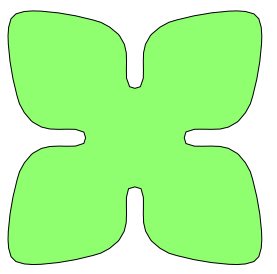

(b)

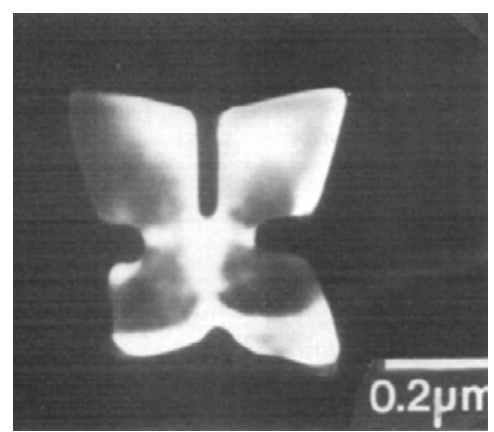

(e)

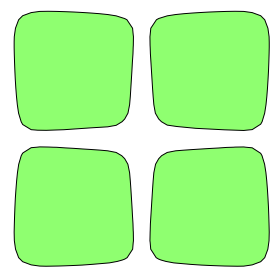

(c)

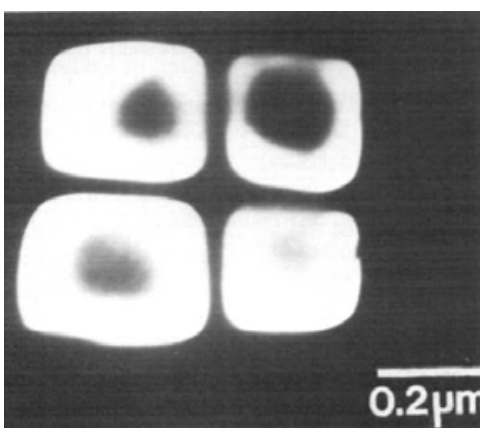

(f)

Fig. 8 Temporal evolution of a coherent particle during quadlet splitting. (a)-(c) are simulation results ( $L=50)$ and (d)-(f) are experimental results (Doi et al., 1984).

Fig. 9 and Fig. 10 show the equilibrium morphologies for different sized particles and the corresponding energy variation curves, respectively. We can see that the splitting behavior does not occur for small size particles. As shown in Fig. 9a, the square-like shape with round corners in Fig. 3b is exactly reproduced for $L=10$, although different initial configurations are used in these two examples. The interfacial energy in this case plays an important role. As a result, it pulls the initial shape back to a square-like one to reduce the interface area. When the particle size becomes larger, the elastic energy gradually dominates and finally leads to particle splitting at a specific size as observed in Fig. 8. In Fig. 10, a steep energy jump for $L=50$ can be clearly 
seen during the evolution, which corresponds to the topological change (splitting) and the total system energy is decreased approximately by $10 \%$ in this process. For the intermediate sized particle, splitting cannot be completed as shown in Fig. 9b, because the elastic energy in this case is not large enough to make it occur.

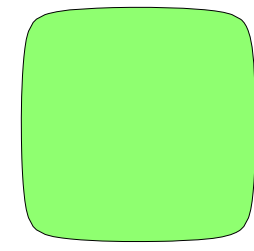

(a)

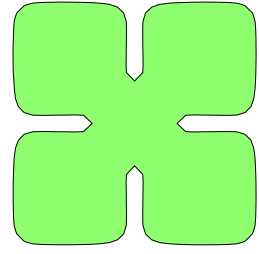

(b)

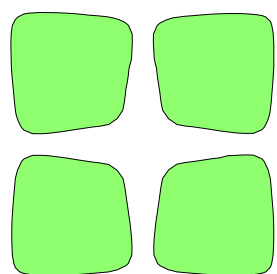

(c)

Fig. 9 Equilibrium morphologies of a four-star shaped particle at different length scales. (a) $L=10$, (b) $L=30$, (c) $L=50$.

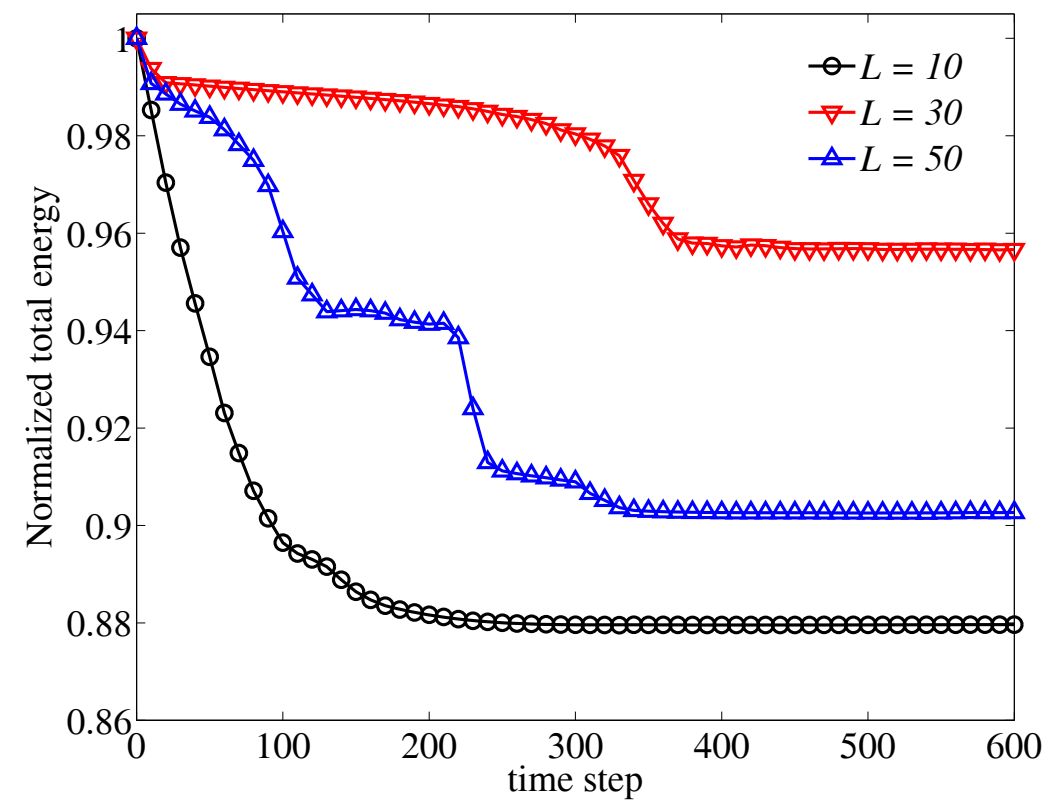

Fig. 10 Energy evolution curves of a star shaped particle for different sizes.

Doublet splitting is another type of characteristic morphology observed in cubic structured alloys. Different from the quadlet splitting above, the disordered $\gamma$ phase 
can nucleate either in the center or at the facets of $\gamma^{\prime}$ cubic and dissolve along the midplane $\{100\}$ direction. Finally, one single square particle splits into two smaller parallel plates. Following the experimental observations (Kaufman et al., 1989), we placed a concave-shaped gap at the top edge of a square particle as the initial configuration in the simulation. Fig. 11 shows the temporal morphological transformation process for $L=50$. It is seen that the concave keeps extending along the $\{100\}$ midplane until it reaches the bottom surface. After splitting, the two parallel rods continue to adjust their shapes and intercenter distance to reduce the elastic energy. It seems that the two parallel plates repel each other at this stage as shown in Fig. 11(d)-(h). Finally they reach an equilibrium morphology as shown in Fig. 11h. This process again agrees remarkably well with experimental observations (Kaufman et al., 1989; Miyazaki et al., 1982). Similar to the quadlet splitting, the doublet splitting is also a result of the elastic energy and does not happen for small particles. Our numerical experiments confirmed that the square-like shape in Fig. 3b and Fig. 9a will be recovered when $L=10$. The energy evolution curve is plotted in Fig. 12, which is similar to the former examples.

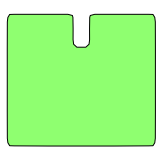

(a)

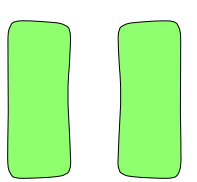

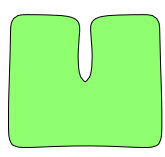

(b)

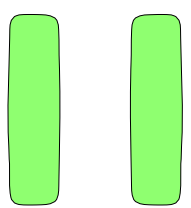

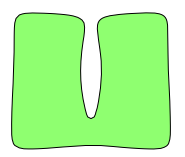

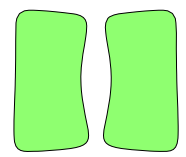

(c)

(d)

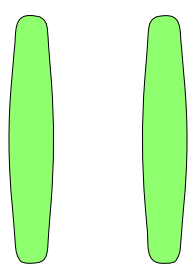

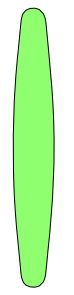


(e)

(f)

(g)

(h)

Fig. 11 Temporal morphological evolution in alloys that undergo a doublet splitting ( $L=50)$. (a)-(h) correspond to the time step 0, 50, 100, 150, 300, 500, 800 and 1200, respectively.

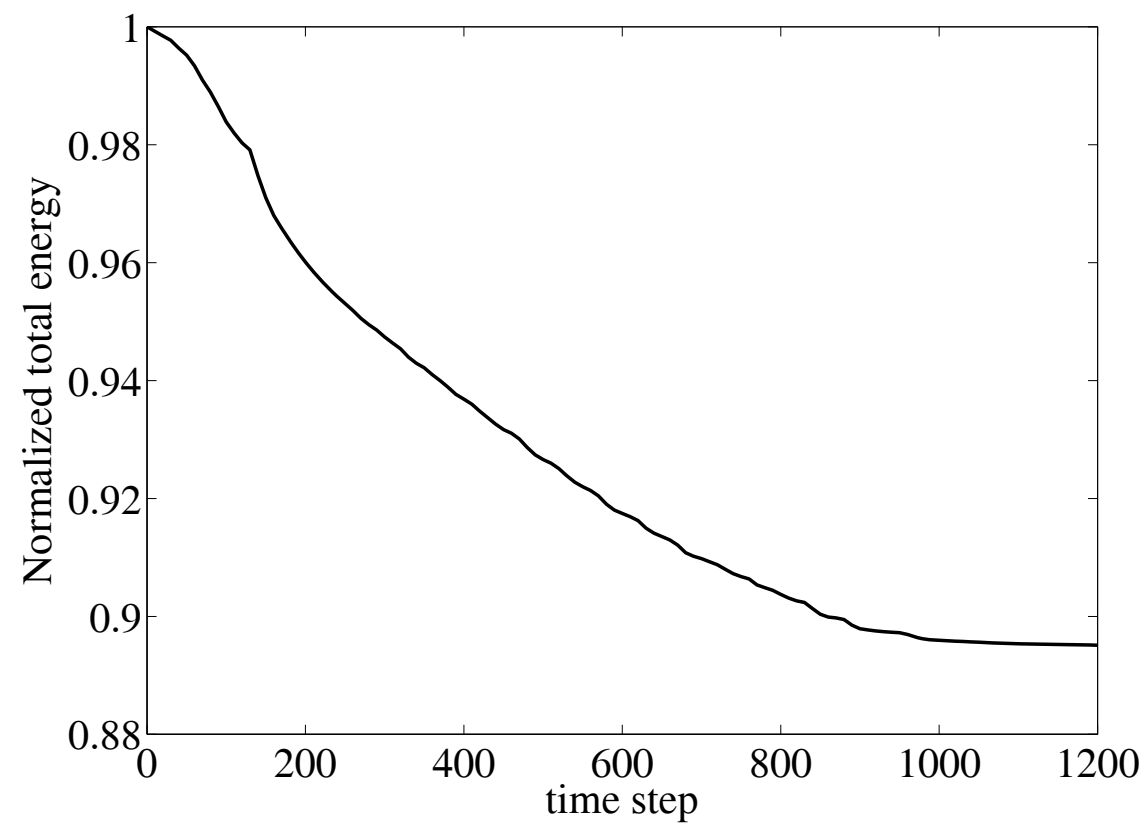

Fig. 12 Energy evolution curve for a coherent particle undergoing doublet splitting

$$
(L=50) \text {. }
$$

\section{Conclusion}

In this paper, a sharp interface model has been developed to study equilibrium morphologies of an inhomogeneity-matrix system that includes the interfacial energy and elastic anisotropy. The driving force on the material interface is derived based on a material derivative approach. The formulation involves using the smoothed extended finite element to calculate the elastic field and the level set method to describe the moving material interface. In such a framework, complex morphological transformations of precipitate particles can be easily captured. Furthermore, the finite element mesh is independent of the interface geometry during the evolution and no remeshing is required, which provides great flexibility for the simulation. 
Numerical results are carried to illustrate the striking influences of elastic energy and interfacial energy on the equilibrium morphologies for particles of different sizes. It is found that small particles tend to favor circle-like shape to minimize the interfacial energy, while large particles exhibit a variety of more complex equilibrium morphologies due to the influence of elastic energy. For multiple particles, their elastic interaction will result in alignment of particles along certain crystallographic directions. When the matrix nucleates at the edges of particles, misfit strain-driven splitting may take place to reduce the total system energy. All our numerical results are in excellent agreement with experiments. Finally, we would like to mention that our simulation here is limited to two dimensions. Also nucleation mechanism is not considered in the present work. Further efforts on extension to three dimensions are being undertaken.

\section{Acknowledgement}

The work presented here was supported in part by NSF through CMMI-1200075. Discussions with Professor David. Chopp on the implementation of the level set and fast marching methods are gratefully acknowledged. 


\section{Reference}

Adalsteinsson, D., Sethian, J.A., 1999. The fast construction of extension velocities in level set methods. J Comput Phys 148, 2-22.

Ardell, A.J., 1968. An Application of Theory of Particle Coarsening - Gamma' Precipitate in Ni-Al Alloys. Acta Metallurgica 16, 511-516.

Belytschko, T., Liu, W.K., Moran, B., 2000. Nonlinear finite elements for continua and structures. John Wiley, Chichester; New York.

Belytschko, T., Moes, N., Usui, S., Parimi, C., 2001. Arbitrary discontinuities in finite elements. Int J Numer Meth Eng 50, 993-1013.

Bordas, S., Nguyen, P.V., Dunant, C., Guidoum, A., Nguyen-Dang, H., 2007. An extended finite element library. Int J Numer Meth Eng 71, 703-732.

Cammarata, R.C., Sieradzki, K., 1994. Surface and Interface Stresses. Annu Rev Mater Sci 24, 215-234.

Chen, S., Merriman, B., Osher, S., Smereka, P., 1997. A simple level set method for solving Stefan problems. J Comput Phys 135, 8-29.

Conley, J.G., Fine, M.E., Weertman, J.R., 1989. Effect of Lattice Disregistry Variation on the Late Stage Phase-Transformation Behavior of Precipitates in Ni-Al-Mo Alloys. Acta Metallurgica 37, 1251-1263.

Dingreville, R., Qu, J.M., Cherkaoui, M., 2005. Surface free energy and its effect on the elastic behavior of nano-sized particles, wires and films. J Mech Phys Solids 53, 1827-1854.

Doi, M., 1996. Elasticity effects on the microstructure of alloys containing coherent precipitates. Prog Mater Sci 40, 79-180.

Doi, M., Miyazaki, T., Wakatsuki, T., 1984. The Effect of Elastic Interaction Energy on the Morphology of Gamma'-Precipitates in Nickel-Based Alloys. Mater Sci Eng 67, 247-253.

Duddu, R., Bordas, S., Chopp, D., Moran, B., 2008. A combined extended finite element and level set method for biofilm growth. Int J Numer Meth Eng 74, 848-870.

Duddu, R., Chopp, D.L., Moran, B., 2009. A Two-Dimensional Continuum Model of Biofilm Growth Incorporating Fluid Flow and Shear Stress Based Detachment. Biotechnol Bioeng 103, 92-104.

Duddu, R., Chopp, D.L., Voorhees, P., Moran, B., 2011. Diffusional evolution of precipitates in elastic media using the extended finite element and the level set methods. J Comput Phys 230, 1249-1264.

Eshelby, J.D., 1975. Elastic Energy-Momentum Tensor. J Elasticity 5, 321-335.

Fahrmann, M., Fratzl, P., Paris, O., Fahrmann, E., Johnson, W.C., 1995. Influence of Coherency Stress on Microstructural Evolution in Model Ni-Al-Mo Alloys. Acta Metall Mater 43, 1007-1022.

Fahrmann, M., Hermann, W., Fahrmann, E., Boegli, A., Pollock, T.M., Sockel, H.G., 1999. Determination of matrix and precipitate elastic constants in (gamma-gamma ') Ni-base model alloys, and their relevance to rafting. Mat Sci Eng a-Struct 260, 212-221.

Gurtin, M.E., Murdoch, A.I., 1975. Continuum Theory of Elastic-Material Surfaces. Archive for Rational Mechanics and Analysis 57, 291-323.

Hu, S.Y., Chen, L.Q., 2001. A phase-field model for evolving microstructures with strong elastic inhomogeneity. Acta Mater 49, 1879-1890.

Jog, C.S., Sankarasubramanian, R., Abinandanan, T.A., 2000. Symmetry-breaking transitions in equilibrium shapes of coherent precipitates. J Mech Phys Solids 48, 2363-2389.

Kaufman, M.J., Voorhees, P.W., Johnson, W.C., Biancaniello, F.S., 1989. An Elastically Induced Morphological Instability of a Misfitting Precipitate. Metall Trans A 20, 2171-2175. 
Liu, G.R., Dai, K.Y., Nguyen, T.T., 2007. A smoothed finite element method for mechanics problems. Comput Mech 39, 859-877.

Liu, X.D., Osher, S., Chan, T., 1994. Weighted Essentially Nonoscillatory Schemes. J Comput Phys 115, 200-212.

Lou, Y., Bassani, J.L., 2008. Guided assembly of nanostructures via elastic interactions. J Mech Phys Solids 56, 3507-3526.

Ma, Y., Ardell, A.J., 2007. Coarsening of gamma (Ni-Al solid solution) precipitates in a gamma' (Ni3Al) matrix. Acta Mater 55, 4419-4427.

Maheshwari, A., Ardell, A.J., 1993. Morphological Evolution of Coherent Misfitting Precipitates in Anisotropic Elastic Media. Phys Rev Lett 70, 2305-2308.

Miyazaki, T., Imamura, H., Kozakai, T., 1982. The Formation of Gamma'-Precipitate Doublets in Ni-Al Alloys and Their Energetic Stability. Mater Sci Eng 54, 9-15.

Moës, N., Cloirec, M., Cartraud, P., Remacle, J.F., 2003. A computational approach to handle complex microstructure geometries. Comput Method Appl M 192, 3163-3177.

Moës, N., Dolbow, J., Belytschko, T., 1999. A finite element method for crack growth without remeshing. Int J Numer Meth Eng 46, 131-150.

Osher, S., Fedkiw, R.P., 2003. Level set methods and dynamic implicit surfaces. Springer, New York.

Osher, S., Sethian, J.A., 1988. Fronts Propagating with Curvature-Dependent Speed - Algorithms Based on Hamilton-Jacobi Formulations. J Comput Phys 79, 12-49.

Povstenko, Y.Z., 1993. Theoretical Investigation of Phenomena Caused by Heterogeneous Surface-Tension in Solids. J Mech Phys Solids 41, 1499-1514.

Provatas, N., Elder, K., 2010. Phase-field methods in materials science and engineering. Wiley-VCH, Weinheim.

Schmidt, I., Gross, D., 1997. The equilibrium shape of an elastically inhomogeneous inclusion. J Mech Phys Solids 45, 1521-1549.

Schmidt, I., Mueller, R., Gross, D., 1998. The effect of elastic inhomogeneity on equilibrium and stability of a two particle morphology. Mech Mater 30, 181-196.

Sethian, J.A., 1996. A fast marching level set method for monotonically advancing fronts. P Natl Acad Sci USA 93, 1591-1595.

Sethian, J.A., 1999a. Fast marching methods. Siam Rev 41, 199-235.

Sethian, J.A., 1999b. Level set methods and fast marching methods : evolving interfaces in computational geometry, fluid mechanics, computer vision, and materials science, 2nd ed. Cambridge University Press, Cambridge, U.K. ; New York.

Shu, C.W., Osher, S., 1988. Efficient Implementation of Essentially Non-Oscillatory Shock-Capturing Schemes. J Comput Phys 77, 439-471.

Shuttleworth, R., 1950. The Surface Tension of Solids. P Phys Soc Lond A 63, 444-457.

Su, C.H., Voorhees, P.W., 1996. The dynamics of precipitate evolution in elastically stressed solids .1. Inverse coarsening. Acta Mater 44, 1987-1999.

Thompson, M.E., Su, C.S., Voorhees, P.W., 1994. The Equilibrium Shape of a Misfitting Precipitate. Acta Metall Mater 42, 2107-2122.

Tian, L., Rajapakse, R.K.N.D., 2007. Analytical solution for size-dependent elastic field of a nanoscale circular inhomogeneity. J Appl Mech-T Asme 74, 568-574.

Voorhees, P.W., Mcfadden, G.B., Johnson, W.C., 1992. On the Morphological Development of 2nd-Phase Particles in Elastically-Stressed Solids. Acta Metall Mater 40, 2979-2992. 
Wang, Y., Chen, L.Q., Khachaturyan, A.G., 1993. Kinetics of Strain-Induced Morphological Transformation in Cubic Alloys with a Miscibility Gap. Acta Metall Mater 41, 279-296.

Zabaras, N., Ganapathysubramanian, B., Tan, L.J., 2006. Modelling dendritic solidification with melt convection using the extended finite element method. J Comput Phys 218, 200-227.

Zhao, X.J., Bordas, S.P.A., Qu, J., 2012. A hybrid smoothed extended finite element/level set method for modeling equilibrium shapes of nano-inhomogeneities. Submitted. 